'Universidad Andres Bello. Facultad de Enfermería. Santiago, Chile. aEnfermera ${ }^{\mathrm{b}} \mathrm{PhD}$.

Trabajo no recibió financiamiento. Las autoras declaran no tener conflictos de interés.

Recibido el 9 de agosto de 2018, aceptado el 17 de enero de 2020.

Correspondencia a: Naldy Febré Vergara Sazié 2212, Santiago Centro. Chile. Naldy.febre@unab.cl

\section{Uso de abreviaturas inseguras en la prescripción médica y errores de medicación: una revisión narrativa}

\author{
KATHERINE MONDACA-GÓMEZ ${ }^{1, a, b}$, NALDY FEBRÉ VERGARA ${ }^{1 a, b}$
}

\section{Unsafe abbreviations in medical prescriptions and their association with medication errors: a review}

The prescription of medications is a weak link in the therapeutic chain of a drug. Medication errors can be associated with the use of so-called unsafe abbreviations in medical prescription. When they are mistakenly interpreted by the person who reads the prescription, error becomes imminent. We herein review the literature about the use of unsafe abbreviations in medical prescriptions and their association with medication errors. PubMed and Lilacs were reviewed, and 29 studies were analyzed, extracting information about the use of abbreviations, common abbreviations, reasons for their use and tools to reduce this conduct. A list with the main risky health abbreviations adapted to Chile was generated. It became clear that the use of abbreviations has increased, appearing in almost all medical prescriptions. The most commonly used abbreviations are the acronym " $u$ " referring to units, followed by "sc" referring to subcutaneous. One of the causes for the use of acronyms the limited time that doctors have at the time of prescription. One way to reduce the use of these abbreviations, is to remind constantly about those that may have the greatest risk in each health institution. A list of unsafe abbreviations, to be used by Chilean health services, is proposed.

(Rev Med Chile 2020; 148: 842-848)

Key words: Medication Errors; Patient Safety; Prescriptions.

\section{I}

os errores de medicación están presentes en la atención de pacientes y causan un gran La prescripción de medicamentos constituye un punto débil dentro de la cadena terapéutica de un fármaco, generando cifras de errores de medicación que superan $50 \%{ }^{1}$, lo que se asocia al uso de las llamadas "abreviaturas inseguras" al momento de la prescripción médica ${ }^{2}$. Las abreviaturas inseguras es la utilización de acrónimos que propensan a errores, al ser interpretados equívocamente por quién los lee ${ }^{3}$. El $77 \%$ de los pacientes hospitalizados poseen una o más abreviaturas inseguras en la prescripción de sus recetas médicas, de las cuales $30 \%$ son consideradas como siglas con alto riesgo de causar daño ${ }^{4}$.

Diferentes organismos nacionales e internacionales abogan por la disminución en la utilización de abreviaturas en salud ${ }^{4,5}$, sin embargo, estas siguen siendo empleadas por los profesionales debido a que facilitan la rapidez de la escritura y permiten tener una utilización más eficaz del tiempo ${ }^{2,6}$.

El objetivo de este estudio es realizar una revisión narrativa en relación al uso de abreviaturas inseguras en la prescripción médica y su asociación con errores de medicación, con el propósito de recomendar una lista de abreviaturas de alto riesgo, factible de aplicar en los servicios sanitarios del país. 


\section{Material y Método}

Para la revisión narrativa fueron seleccionadas las siguientes bases de datos: PubMed, y Lilacs. Estas fueron escogidas debido a que poseían los recursos más completos en salud de la literatura mundial, latinoamericana y del caribe.

La búsqueda en la base de datos empleó los siguientes descriptores: "Abbreviations as Topic"[Mesh] AND ("Medication Errors"[Mesh] OR "Inappropriate Prescribing"[Mesh]). Se utilizaron los recursos de información bibliográfica de la Universidad Andrés Bello para la extracción de los artículos, según estrategia de búsqueda.

Se tuvo acceso a 124 artículos, de los cuales 23 fueron excluidos por no tener acceso a texto completo, 63 por no tener relación con la temática y 9 por estar repetidos en la base de datos, por lo que se escogieron y analizaron 29 artículos (Figura 1). Las secciones utilizadas para el análisis de los estudios fueron: Métodos, Resultados y Conclusiones. Con estos apartados, se efectuó un análisis de contenido, que incluyó los siguientes aspectos: utilización de abreviaturas en la prescripción médica, abreviaturas comúnmente utilizadas, causas del uso de abreviaturas en salud y herramientas para disminuir su utilización. Además de ello, se redactó un listado con las principales abreviaturas de alto riesgo en salud adaptadas a Chile, donde se especificó la abreviatura, su significado, la interpretación errónea que puede generar y como debe expresarse correctamente.

\section{Resultados}

Posterior al análisis de los artículos seleccionados, los resultados fueron agrupados de la siguiente manera:

\section{Utilización de abreviaturas en la prescripción médica}

El uso de abreviaturas confusas o de fácil mal interpretación pusieron en riesgo a los cuidadores de cometer errores comprometiendo la seguridad de los pacientes. Una mal interpretación pudo tener resultados catastróficos, confundiendo por

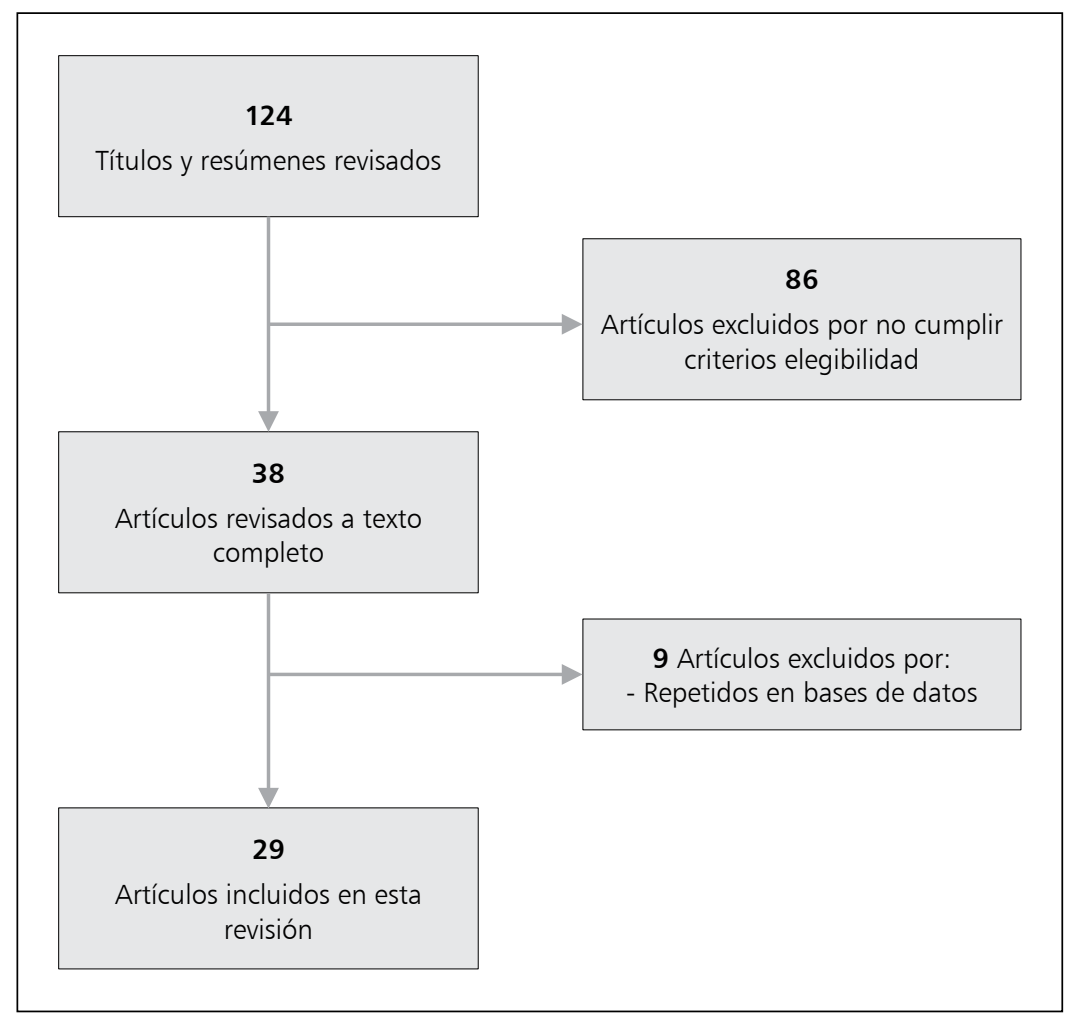

Figura 1. Flujograma de inclusión y exclusión de artículos. 
ejemplo, "U” con un "0 (cero)" "7,8. Expertos señalaron que desde el año 2004, el Institute for Safe Medication Practices ISMP recomendó una lista de abreviaturas de alto riesgo que incluía acrónimos, símbolos y designaciones de dosis que no debían ser utilizadas de ninguna forma en el sector sanitario9. Así también, The Join Commision emitió una nómina con similares características. El objetivo de ambas instituciones, fue reducir el número de errores de medicación asociados a la presencia de acrónimos en salud ${ }^{10,11}$, debido a que fue posible que se generaran errores de medicación mediante el uso de abreviaturas ${ }^{12,13}$.

El año 2007, The Join Commision difundió un comunicado oficial, en donde explicitó el impacto del uso de abreviaturas en la seguridad de los pacientes. En él, se dió a conocer que desde el año 2004 hasta el año 2006 del total de errores de medicación reportados, $4,7 \%$ de ellos eran atribuibles al uso de abreviaturas ${ }^{11}$.

Además de lo anterior, existe evidencia proviniente de estudios realizados entre los años 2008 y 2011 en Brasil, que concluyó que la prevalencia de siglas y/o abreviaturas en las prescripciones médicas tenían relación con los errores de medicación, en donde se revisó los errores en el horario de administración y en esa muestra encontró 96,2\% de existencia de abreviaturas ${ }^{14}$, y $93,8 \%$ de las prescripciones que poseían abreviaturas se relacionaban con errores en la vía de administración de los fármacos ${ }^{14,15}$.

En relación a la frecuencia del uso de abreviaturas en la prescripción médica, fueron diversos los estudios que abordaron el tema, y en todos ellos se observaron cifras mayores a $77 \%$, las que aumentaron con el pasar de los años hasta llegar a $100 \%$ en el año $2013^{4,16}$.

\section{Abreviaturas comúnmente utilizadas en las prescripciones médicas}

Un estudio efectuado en Sri Lanka señaló que existieron 49 tipos de abreviaturas incorporadas en la lista recomendada por el ISMP ${ }^{2}$, no obstante, otros autores describieron 70 tipos diferentes de siglas, encontrando una media de 33,3 abreviaturas por prescripción ${ }^{17}$.

Dentro de las abreviaturas que más se repitieron, correspondieron principalmente al uso de: " $u$ " o "iu” para unidades, "sc" para subcutáneo, "comp" para comprimido, " $x$ " para número de veces, "vo" para vía oral, "iv/ev" para introvenoso/ endovenoso, "im" para intramuscular, "SF" para suero fisiológico, " $m g$ ” para miligramo, " $h$ " para hora y "gts" para gotas ${ }^{11,16,18,19}$.

Además de ello, existieron distintos tipos de abreviaturas, donde se distinguieron acrónimos de bajo y de alto riesgo. Los de alto riesgo se refirieron a las abreviaturas contenidas en la lista que emitió el ISMP. Según un estudio realizado en Arabia Saudita el año 2016, la incidencia del uso de abreviaturas de alto riesgo en la prescripción médica correspondió a $72,7 \%{ }^{20}$, se trató de abreviaturas que pudieron causar consecuencias mortales en los pacientes, tales como " $и$ o iu", las que pudieron ser erróneamente confundidas por un 0 o por un 4 , con riesgo de multiplicar por 10 o más la dosis (por ejemplo, $4 \mathrm{U}$ pudo haberse confundido con $40)^{21}$. Autores señalaron, que independiente si las abreviaturas eran de alto o bajo riesgo, éstas debían ser evitadas ${ }^{4}$.

Paparella señaló que el medicamento que generó más errores de medicación fue la insulina, donde el evento adverso más reportado fue la dosis inadecuada, generando hipoglicemia o hiperglicemia ${ }^{22}$.

\section{Causas del uso de abreviaturas en salud}

El hábito de acortar palabras fue parte del lenguaje de salud, con el objetivo de ahorrar tiempo y ser más eficientes, de forma que se pudieron encontrar abreviaturas en casi todas las órdenes médicas, sean éstas manuales o electrónicas ${ }^{23}$. Existe evidencia que señaló que la utilización de abreviaturas en salud era considerada como una forma de economizar tiempo, debido a que comúnmente era un único médico el responsable de prescribir indicaciones para múltiples pacientes $^{11,14}$, además de ello, los encargados señalaron que las prescripciones eran realizadas bajo un ambiente de interrupciones y distracciones ${ }^{24}$. Aunque el uso de abreviaturas pudo aparecer inicialmente para ahorrar tiempo, pudo causar un gran retraso para aquellos que debían interpretarlas ${ }^{25}$.

Sumado a lo previo, existió consenso entre los profesionales prescriptores, de que la utilización de abreviaturas en su trabajo diario economizaba su tiempo, no obstante, estaban en conocimiento de que éstas podían ser mal interpretadas o podían tener más de un significado para los lectores. Además de ello, los profesionales médicos asumieron desconocer las abreviaturas que podían causar un gran daño al paciente $e^{11,15,20,24}$. 
Un estudio efectuado en Pakistán clarificó que la interpretación de abreviaturas en prescripciones médicas, dependió directamente del conocimiento y de la experiencia de quién las leyó ${ }^{21}$. Así también, autores indicaron que el proceso de medicación era frágil en relación a los riesgos para la seguridad del paciente, necesitando de manera urgente la implantación de medidas preventivas y correctivas para todo el equipo involucrado en la terapia farmacológica ${ }^{16}$, y es que el uso de abreviaturas contribuyó a errores cuando se mal interpretó un símbolo o una letra, y esta mal interpretación generó que se administrara un medicamento o dosis incorrecta, o se diera el medicamento en el momento equivocado o con la frecuencia incorrecta $^{26}$.

\section{Herramientas para disminuir el uso de abrevia- turas en salud}

Un estudio efectuado en Arabia Saudita señaló que la ejecución de intervenciones educativas por parte de un Farmacéutico disminuyó significativamente la incidencia de abreviaturas de alto riesgo en salud $(53,6 \% \text { vs } 25,5 \% ; p=0,001)^{20}$. Así también, existe evidencia de que la responsabilidad de la terapia farmacológica era multidisciplinaria, por lo que resultaba necesario que la prescripción no fuese vista a penas como un documento, sino más bien, como un instrumento de comunicación entre los profesionales ${ }^{16,24}$.

Autores señalaron que aun cuando muchos hospitales tenían incorporada la lista de "no utilizar" recomendada por el ISMP, era necesario actualizarla periódicamente, y recordar su existencia de manera frecuente 9 . Sumado a lo anterior, investigadores plantearon que el uso de alertas computarizadas para recordar la lista de abreviaturas no recomendadas, mostró una reducción significativa en el empleo de abreviaturas, incluso cuando éstas eran escritas a mano ${ }^{19}$.

Así también, existe evidencia de que la utilización de prescripción electrónica no erradicó la posibilidad de errores de medicación, una vez que ésta permitió el uso de siglas y abreviaturas ${ }^{15,27}$. Además de ello, estudios señalaron que era necesario que los profesionales fueran continuamente entrenados en el sentido de evitar el empleo de acrónimos en las prescripciones, inclusive cuando éstas se encontraban estandarizadas en las instituciones sanitarias ${ }^{4,15}$, el uso de abreviaturas es arriesgado y debería ser evitado. Las abreviaturas son una forma de código, y los códigos sólo son efectivos cuando el emisor y el receptor han acordado usarlos ${ }^{28}$.

\section{Listado con las principales abreviaturas de alto riesgo en salud}

El análisis de los 29 artículos, sumado a la lista de "no utilizar" del ISMP posibilitó generar un listado con las principales abreviaturas de alto riesgo en salud adaptadas a Chile, en donde se explicitó el nombre de la abreviatura, su significado, la interpretación errónea que puede adquirir y la forma en la que debe expresarse correctamente. La adaptación a Chile consistió en traducir los términos y seleccionar los más utilizados en nuestro país (Tabla 1).

\section{Discusión}

Esta revisión de literatura permite describir la frecuencia de utilización de abreviaturas en la prescripción médica, abreviaturas comúnmente utilizadas, causas del uso de abreviaturas en salud y herramientas para disminuir su utilización. Además de ello, recomienda una lista de abreviaturas de alto riesgo, factible de aplicar en los servicios sanitarios del país, adaptada del ISMP.

Los errores de medicación asociados al uso de abreviaturas en salud ocurren cada día en miles de hospitales a nivel mundial ${ }^{3}$, algunos de ellos han intentado contrarrestar el empleo de abreviaturas, utilizando, por ejemplo, fichas clínicas electrónicas, no obstante, autores demuestran que es una medida que no reduce la frecuencia de uso de abreviaturas, y que, al permitir el empleo de éstas, se abre la oportunidad a los errores de medicación ${ }^{15}$. Existen autores que señalan que el uso de abreviaturas, además de generar errores de medicación, arruinan la comunicación al interior de los equipos de salud ${ }^{29}$. Es importante destacar, que el Institute for Safe Medication Practices ISPM establece que las abreviaturas nunca deben ser utilizadas en prescripciones, y que no existe un mecanismo más efectivo que eliminarlas por completo del ambiente clínico ${ }^{4}$. Es por lo anterior, que futuras investigaciones debieran centrarse en crear mecanismos eficaces para eliminar por completo la utilización de abreviaturas en salud, no reducirlas.

Existe consenso entre los autores, que las abreviaturas más utilizadas son " $U$ " y " $s c$ ", las cuales 
Tabla 1. Principales abreviaturas de alto riesgo en salud adaptadas a Chile* (ajustada de referencia 2)

\begin{tabular}{|c|c|c|c|}
\hline Abreviaturas** & Significado & Malinterpretación & Expresión correcta \\
\hline $\mathrm{HCT}$ & Hidrocortisona & Confusión con "Hidroclorotiazida". & $\begin{array}{l}\text { Usar el "nombre completo del medi- } \\
\text { camento" }\end{array}$ \\
\hline MTX & Metotrexato & Confusión con "Mitoxantrona". & $\begin{array}{l}\text { Usar el "nombre completo del medi- } \\
\text { camento" }\end{array}$ \\
\hline $\mathrm{cc}$ & $\begin{array}{l}\text { Centímetro } \\
\text { cúbico }\end{array}$ & $\begin{array}{l}\text { Confusión con "0" o con "u" (uni- } \\
\text { dades). }\end{array}$ & Usar "mL" \\
\hline U o u & Unidades & $\begin{array}{l}\text { Confusión con "0" o "4", con riesgo } \\
\text { de multiplicar por } 10 \text { o más la dosis }\end{array}$ & Usar "unidades" \\
\hline$d$ & Día & $\begin{array}{l}\text { Confusión con dosis ( } 3 \text { d para indicar } \\
3 \text { días, puede confundirse con } 3 \\
\text { dosis) }\end{array}$ & Usar "días" \\
\hline IN & Intranasal & Confusión con "IM" o "IV" & Usar "intranasal" \\
\hline SC & Subcutáneo & Confusión con "SL" (sublingual). & Usar "subcutáneo" \\
\hline IU & $\begin{array}{l}\text { Unidades } \\
\text { internacionales }\end{array}$ & $\begin{array}{l}\text { Confusión con "IV" (intravenoso) o } \\
\text { "10" }\end{array}$ & Usar "unidades" \\
\hline+ & Más o y & Confusión con "4" & Usar "y" \\
\hline$x$ & Durante & $\begin{array}{l}\text { Confusión con "cada" ("x } 2 \text { días" } \\
\text { puede interpretarse como "cada } 2 \\
\text { días", en vez de "durante } 2 \text { días". }\end{array}$ & Usar "durante" \\
\hline$>_{1}<$ & $\begin{array}{l}\text { Mayor que y } \\
\text { menor que }\end{array}$ & $\begin{array}{l}\text { Confusión entre los dos símbolos. } \\
\text { Además "< } 10 \text { " puede confundirse } \\
\text { con " } 40 \text { " }\end{array}$ & Usar "mayor que" y "menor que" \\
\hline $\begin{array}{l}\text { Usar el cero detrás de la } \\
\text { coma decimal (1,0 mg) }\end{array}$ & $1 \mathrm{mg}$ & $\begin{array}{l}\text { Confusión con "10 mg" si la coma } \\
\text { no se ve bien }\end{array}$ & $\begin{array}{l}\text { Si la dosis se expresa con números } \\
\text { enteros, no se debe poner cero } \\
\text { detrás de la coma }\end{array}$ \\
\hline $\begin{array}{l}\text { Escribir juntos el nombre } \\
\text { del fármaco y la dosis } \\
\text { (Propanolol20 mg) }\end{array}$ & $\begin{array}{l}\text { Propanolol } 20 \\
\text { mg }\end{array}$ & Confusión con "propanolol 120 mg" & $\begin{array}{l}\text { Dejar suficiente espacio entre el } \\
\text { nombre del fármaco, la dosis y las } \\
\text { unidades de dosificación }\end{array}$ \\
\hline $\begin{array}{l}\text { Escribir juntos la dosis } \\
\text { y las unidades de } \\
\text { dosificación }(10 \mathrm{mg})\end{array}$ & $10 \mathrm{mg}$ & $\begin{array}{l}\mathrm{La} \text { " } \mathrm{m} \text { " se puede confundir con un } \\
\text { cero o dos ceros, con riesgo de mul- } \\
\text { tiplicar por } 100 \text { por } 100 \text { la dosis }\end{array}$ & $\begin{array}{l}\text { Dejar suficiente espacio entre la dosis } \\
\text { y las unidades de dosificación }\end{array}$ \\
\hline $\begin{array}{l}\text { Escribir número grandes } \\
\text { para indicar la dosis, } \\
\text { sin utilizar puntos para } \\
\text { separar los millares } \\
\text { (100.000 unidades) }\end{array}$ & $\begin{array}{l}100.000 \\
\text { unidades }\end{array}$ & $\begin{array}{l}\text { "100000" se puede confundir con } \\
\text { "10.000" o con "1.000.000" }\end{array}$ & $\begin{array}{l}\text { Para números superiores a } 1.000, \\
\text { usar puntos para separar los millares, } \\
\text { o usar expresiones como "1 millón". } \\
\text { No usar "M" para referirse a "millón" } \\
\text { porque puede confundirse con "mil" }\end{array}$ \\
\hline
\end{tabular}

*Estas abreviaturas jamás deben emplearse al comunicar información médica². **Se utilizó como sinónimo de abreviatura a los acrónimos, símbolos y designaciones de dosis.

corresponden a acrónimos de alto riesgo ${ }^{21}$, es decir, son abreviaturas que pueden causar la muerte de un paciente al ser interpretadas equívocamente por quien las lee ${ }^{6}$.

Así también, es importante señalar que los profesionales médicos están conscientes que utilizar abreviaturas en sus prescripciones pueden generar daño, no obstante, las siguen empleando ${ }^{11,15}$.

En relación a las herramientas para disminuir la utilización de abreviaturas en salud, son pocos los estudios que muestran efectividad en sus intervenciones. Se destaca la incorporación 
de un químico farmacéutico dentro del proceso farmacológico como un mecanismo eficaz para disminuir el uso de abreviaturas ${ }^{17}$, no obstante, son acciones que no todos los centros asistenciales pueden costear. Además de ello, autores coinciden en que es de vital importancia que cada establecimiento sanitario tenga su propia lista con abreviaturas que no se deben utilizar, debido a que es una herramienta eficaz, sencilla y de bajo costo que ha demostrado cierta efectividad en la disminución de la utilización de abreviaturas inseguras por parte de los prescriptores, más no las elimina ${ }^{2,4,9,15,24}$. Los esfuerzos a partir de esta revisión de literatura están enfocados a invitar a los establecimientos sanitarios a actualizarse sobre la utilización de abreviaturas y a considerar la incorporación de la lista adjunta como parte de su proceso de prescripción médica.

Una limitación de esta revisión, es el escaso número de artículos relacionados con el tema (29 artículos), de los cuales el más actualizado data del año 2018. Un 14\% de los artículos encontrados con los términos Mesh descritos en material y métodos, correspondió exclusivamente a revisiones de literatura que mostraban ejemplos fotográficos de los tipos de abreviaturas frecuentemente utilizadas.

Dentro de los hallazgos detectados, es posible determinar que la utilización de abreviaturas en salud es una práctica frecuente a nivel mundial, y se asocia a la presencia de errores de medicación. Con el pasar de los años, el aumento de la complejidad en la medicina, sumada a la gran carga laboral de los prescriptores en salud ha conllevado inevitablemente a un aumento en la frecuencia de utilización de acrónimos ${ }^{4,16}$.

\section{Referencias}

1. Taravilla-Cerdan B, Larrubia-Muñoz O, de la Corte-García M, Cruz-Martos E. Tracing the map of medication errors outside the hospital environment in the Madrid Community. Aten Primaria 2011; 43 (12): 648-55.

2. Samaranayake NR, Cheung DS, Lam MP, Cheung TT, Chui WC, Wong IC, et al. The effectiveness of a 'Do Not Use' list and perceptions of healthcare professionals on error-prone abbreviations. Int J Clin Pharm 2014; 36 (5): 1000-6.

3. Otero MO, Muñoz M, Domínuez-Gil H. Seguridad de medicamentos: abreviaturas, símbolos y expresiones de dosis asociados a errores de medicación. Farm Hosp 2004; 28 (2): 141-4.

4. Dooley MJ, Wiseman M, Gu G. Prevalence of error-prone abbreviations used in medication prescribing for hospitalised patients: multi-hospital evaluation. Intern Med J 2012; 42 (3): 19-22.

5. Ministerio de Salud Gobierno de Chile. Guía para las buenas prácticas de prescripción 2010. https://www. minsal.cl/wp-content/uploads/2015/09/2CD_GUIAPARA-LAS-BUENAS.pdf

6. Soto-Arnáez F, Sebastián-Viana T, Carrasco-Garrido P, Fernández de las Peñas $\mathrm{P}$, Palacios-Ceña D. The use of unsafe abbreviations in discharge report and medical prescription: observational and retrospective study. Anales Sis San Navarra 2016; 39 (3): 379-87.

7. Association of Perioperative Registered Nurses. AORN Guidance Statement: "do-not-use" abbreviations, acronyms, dosage designations, and symbols. AORN J. 2006; 84 (3): 489-92.

8. Weaver JM. It's time to throw out old-fashioned latin abbreviations. Anesth Prog 2006; 53 (1): 1-2.

9. Institute for Safe Medication Practices. List of Error-Prone Abbreviations, Symbols, and Dose Designations 2015.

10. Eschenbach von AC. Eliminating error-prone notations in medical communications. Expert Opinion on Drug Safety 2007; 6 (3): 233-4.

11. Brunetti L, Santell JP, Hicks RW. The Impact of Abbreviations on Patient Safety. Joint Commission. Journal on Quality and Patient Safety The Joint Commission 2007; 33 (9): 576-83.

12. Hutton M. Calculations for new prescribers. Nurs Stand 2003; 17 (25): 47-52.

13. Schulmeister L. Look-Alike, Sound-Alike Oncology Medications. Clin J Oncol Nurs 2006; 10 (1): 35-41.

14. Escobar F, Cardoso T, Bauer A, Perufo S, Ludermiller $\mathrm{M}$, De Bertoli S. Influence of the writing of the medical orders on the administration of medications at the wrong schedule time. Acta Paul Enferm 2009; 22 (4): 380-4.

15. Escobar F, Marques T, Cardoso T, Sabóia M, Bauer A, De Bortoli S. Administración de medicamentos en vías diferentes de las indicadas en la prescripción médica. Rev Latino-Am Enfermagem 2011; 19 (1): 1-7.

16. Silva FJCPD, Almeida ESA, Rocha FC, Santana MAV, Silva ECCM, Lemos LMD, et al. Analysis of the records of drug prescriptions in a university hospital. Rev Min Enferm 2015; 19 (3): 1-8.

17. Borges M, Perini E, Azevedo T, Miranda H, Bogutchi T. Erros na prescrição hospitalar de medicamentos 
potencialmente perigosos. Rev Saúde Pública 2009; 43 (3): 490-8.

18. Siqueira JS, Antoniolli AR, Silvestre CC, Oliveira-Filho AD, Silva WB, Lyra DP. Analysis of the quality of prescriptions at a cardiovascular ward in Brazil: a pilot study. Int J Clin Pharm 2011; 33 (2): 260-3.

19. Myers JS, Gojraty S, Yang W, Linsky A, Airan-Javia S, RCP. A randomized-controlled trial of computerized alerts to reduce unapproved medication abbreviation use. J Am Med Inform Assoc 2011; 18 (1): 17-23.

20. Haseeb A, Winit-Watjana W, Bakhsh A-RR, Elrggal ME, Hadi MA, Mously AA. Effectiveness of a pharmacist-led educational intervention to reduce the use of high-risk abbreviations in an acute care setting in Saudi Arabia: a quasi-experimental study. BMJ Open 2016; 6 (6): 401-7.

21. Awan S, Abid S, Tariq M, Zubairi ABS, Kamal A, Arshad S. Use of medical abbreviations and acronyms: knowledge among medical students and postgraduates. Postgrad
Med J 2016; 92 (1094): 721-5.

22. Paparella S. Avoiding Errors With Insulin Therapy. J EMERG NURS 2006; 32 (4): 325-8.

23. Paparella S. Avoiding Dangerous Abbreviations and Dose Expressions. J Emerg Nurs 2004; 30 (1): 54-8.

24. Bauer A, De Bertoli S, Inocenti A, Perufo S. Problemas na comunicacao: uma possível causa de erros de medicacao. Acta Paul Enferm 2007; 20 (3): 272-6.

25. Becker S. Common Causes for Medication Errors Identified. Int J Trauma Nurs 1999; 5(3): 113-5.

26. Suzanne C, Beyea RN. Best practices for abbreviation use. Aorn J 2004; 79 (3): 641-2.

27. Thomas MP. Medication Errors. Clin Pediatr. 2003; 1 (42): 287-94.

28. Pickup J. Cause of dosing errors. Lancet 2003; 362 (19): 252.

29. Walsh KE, Gurwitz JH. Medical abbreviations: writing little and communicating less. Archives of Disease in Childhood 2008; 93 (10): 816-7. 